\title{
A HILBERT-SCHMIDT NORM INEQUALITY ASSOCIATED WITH THE FUGLEDE-PUTNAM THEOREM
}

\author{
TAKAYUKI FURUTA
}

\begin{abstract}
Dedicated in deep sorrow to the memory of the late Professor Teishirô Saitô
\end{abstract}

\begin{abstract}
The familiar Fuglede-Putnam theorem asserts that $A X=X B$ implies $A^{*} X=X B^{*}$ when $A$ and $B$ are normal. We prove that $A$ and $B^{*}$ be hyponormal operators and let $C$ be a hyponormal commuting with $A^{*}$ and also let $D^{*}$ be a hyponormal operator commuting with $B$ respectively, then for every Hilbert-Schmidt operator $X$, the Hilbert-Schmidt norm of $A X D+C X B$ is greater than or equal to the Hilbert-Schmidt norm of $A^{*} X D^{*}+C^{*} X B^{*}$. In particular, $A X D=C X B$ implies $A^{*} X D^{*}=C^{*} X B^{*}$. If we strengthen the hyponormality conditions on $A, B^{*}, C$ and $D^{*}$ to quasinormality, we can relax Hilbert-Schmidt operator of the hypothesis on $X$ to be every operator and still retain the inequality under some suitable hypotheses.
\end{abstract}

\section{Introduction}

An operator means a bounded linear operator on a separable infinite dimensional Hilbert space $H$. Let $B(H)$ and $C_{2}$ denote the class of all bounded linear operators acting on $H$ and the Hilbert-Schmidt class in $B(H)$ respectively. $C_{2}$ forms a two-sided ideal in the algebra $B(H)$ and $C_{2}$ is itself a Hilbert space for the inner product

Received 2 September 1981. 


$$
(X, Y)=\sum\left(X e_{j}, Y_{j}\right)=\operatorname{Tr}\left(Y^{*} X\right)=\operatorname{Tr}\left(X Y^{*}\right)
$$

where $\left\{e_{j}\right\}$ is any orthonormal basis of $H$ and $\operatorname{Tr}(T)$ denotes the trace. In what follows, $\|T\|_{2}$ denotes the Hilbert-Schmidt norm.

An operator $T$ is called quasinormal if $T$ commutes with $T^{*} T$, subyormal if $T$ has a normal extension and hyponormal if $\left[T^{*}, T\right] \geq 0$ where $[S, T]=S T-T S$. The inclusion relation of the classes of nonnormal operators listed above is as follows:

$$
\text { Normal } \varsubsetneqq \text { Quasinormal } \varsubsetneqq \text { Subnormal } \varsubsetneqq \text { Hyponormal ; }
$$

the above inclusions are all proper [6, Problem 160, p. 101].

In [2], Berberian shows the following result.

THEOREM $A$ [2]. If $A$ and $B^{*}$ are hyponormal, then $A X=X B$ implies $A^{*} X=X B^{*}$ for an operator $X$ in the Hilbert-Schmidt class.

On the other hand, in [3] we have shown Theorem B which is an extension of the Fuglede-Putnam theorem.

THEOREM $B$ [3]. If $A$ and $B^{*}$ are subnormal and if $X$ is an operator such that $A X=X B$, then $A^{*} X=X B^{*}$.

Recently Weiss has obtained the following result.

THEOREM $C$ [11]. Let $\left\{A_{1}, A_{2}\right\}$ and $\left\{B_{1}, B_{2}\right\}$ denote commuting pairs of normal operators and let $X \in B(H)$. Then

$$
\left\|A_{1} X B_{1}+A_{2} X B_{2}\right\|_{2}=\left\|A_{1}^{*} X B_{1}^{*}+A_{2}^{*} X B_{2}^{*}\right\|_{2} \text {. }
$$

In this paper we prove Theorem $I$ which is an extension of Theorem A and also we prove a slightly stronger Theorem 2 by integrating Theorem $B$ and Theorem $\mathrm{C}$.

2.

First of all we show the following theorem.

THEOREM 1. Let $A$ and $B^{*}$ be hyponormal on $H$. Let $C$ be $a$ hyponormal commuting with $A^{*}$ and also $D^{*}$ be a hyponormal commuting with $B$ respectively. Then 
(i)

(*)

$$
\|A X D+C X B\|_{2} \geq\left\|A^{*} X D^{*}+C^{*} X B^{*}\right\|_{2}
$$

holds for every $X$ in the Hilbert-Schmidt class. Equality in (*) holds for every $X$ in the Hilbert-Schmidt class when $A, B, C$ and $D$ are all normal.

(ii) If $X$ is an operator in the Hilbert-Schmidt class such that $A X D=C X B$, then $A^{*} X D^{*}=C^{*} X B^{*}$.

Proof. Define an operator $T$ on $C_{2}$ as follows:

$$
T X=A X D+C X B \text {. }
$$

Then, if we view $C_{2}$ as an underlying Hilbert space, then $T^{*}$ exists and $T^{*}$ is given by the formula $T^{*} X=A^{*} X D^{*}+C^{*} X B^{*}$ since we easily see from

$$
\begin{aligned}
\left(T^{*} X, Y\right) & =(X, T Y)=(X, A Y D+C Y B)=\operatorname{Tr}\left(X D^{*} Y^{*} A^{*}\right)+\operatorname{Tr}\left(X B^{*} Y^{*} C^{*}\right) \\
& =\operatorname{Tr}\left(A^{*} X D^{*} Y^{*}\right)+\operatorname{Tr}\left(C^{*} X B^{*} Y^{*}\right)=\operatorname{Tr}\left(\left(A^{*} X D^{*}+C^{*} X B^{*}\right) Y^{*}\right) \\
& =\left(A^{*} X D^{*}+C^{*} X B^{*}, Y\right) .
\end{aligned}
$$

Also

$$
\begin{aligned}
\left(T * T-T T^{*}\right) X= & A^{*}(A X D+C X B) D^{*}+C^{*}(A X D+C X B) B^{*} \\
& -A\left(A^{*} X D^{*}+C^{*} X B^{*}\right) D-C\left(A^{*} X D^{*}+C^{*} X B^{*}\right) B \\
= & \left(A^{*} A X D D^{*}-A A^{*} X D^{*} D\right)+\left(C^{*} C X B B^{*}-C C^{*} X B^{*} B\right) \\
& +A^{*} C X B D^{*}-A C^{*} X B^{*} D+C^{*} A X D B^{*}-C A^{*} X D^{*} B \\
= & \left(A^{*} A-A A^{*}\right) X D D^{*}+A A^{*} X\left(D D^{*}-D^{*} D\right)+\left(C^{*} C-C C^{*}\right) X B B^{*}+C C^{*} X\left(B B^{*}-B^{*} B\right) \\
& +\left(A^{*} C X B D^{*}-C A^{*} X D^{*} B\right)+\left(C^{*} A X D B^{*}-A C^{*} X B^{*} D\right)
\end{aligned}
$$

and the fifth and the sixth terms in the formula above are both zero since the hypotheses $C A^{*}=A^{*} C$ and $D^{*} B=B D^{*}$ hold, so that

(1) $\left(T^{*} T-T T^{*}\right) X=\left(A^{*} A-A A^{*}\right) \cdot X D D^{*}+A A^{*} X\left(D D^{*}-D^{*} D\right)$

$$
+\left(C^{*} C-C C^{*}\right) X B B^{*}+C C^{*} X\left(B B^{*}-B^{*} B\right) \text {. }
$$

Left and right multiplication acting on $C_{2}$ as the Hilbert space by a positive operator is itself a positive operator. Since $T * T-T^{*}$ is the sum of four positive operators by the hyponormality of $A, B^{*}, C$ and $D^{*}$, $T$ is hyponormal. Therefore

$$
\|T X\|_{2} \geq\|T * X\|_{2}
$$


that is,

$$
\|A X D+C X B\|_{2} \geq\left\|A^{*} X D^{*}+C^{*} X B^{*}\right\|_{2}
$$

and the proof of equality easily follows by (1) and (2). If an operator $T$ is hyponormal, then $-T$ is also hyponormal, so the proof of (ii) easily follows by (*) in (i).

COROLLARY 1. Let $A$ and $B^{*}$ be hyponormal on $H$. Let $C$ be $a$ normal commuting with $A$ and also let $D$ be a normal commuting with $B$ respectively. Then

(i)

$$
\|A X D+C X B\|_{2} \geq\left\|A^{*} X D^{*}+C^{*} X B^{*}\right\|_{2}
$$

holds for every $X$ in the Hilbert-Schmidt class. Equality in (*) holds for every $X$ in the Hilbert-Schmidt class when $A$ and $B$ are both normal.

(ii) If $X$ is an operator in the Hilbert-Schmidt class such that $A X D=C X B$, then $A^{*} X D^{*}=C^{*} X B^{*}$.

Proof. The hypotheses $C A=A C$ and $D B=B D$ imply $C A^{*}=A^{*} C$ and $D B^{*}=B^{*} D$, that is, $D^{*} B=B D^{*}$ by the original Fuglede--Putnam theorem $[1],[6],[7],[8]$, so the proof follows by Theorem 1 .

REMARK 1. We remark that Weiss [10, Theorem 4] shows the case of the equality in $(i)$ of Corollary $I$ when $A=B$ is normal and $C=D=I$ the identity operator on $H$, by a different method and also Corollary $I$ is an extension of Theorem A.

3.

If we strengthen the hyponormality conditions to quasinormality, then we can relax the Hilbert-Schmidt operator of the hypothesis on $X$ to be every operator in $B(H)$ in Theorem $I$ and still retain the inequality under some suitable hypotheses.

DEFINITION 1. Let $N_{T}$ denote a normal extension on $H \oplus H$ of a subnormal operator $T$ on $H$. In fact, for every subnormal operator $T$, there exists a normal extension $N_{T}$ on $H \oplus H$ whose restriction to $H \oplus\{0\}$ is $T$ [5]. 
LEMMA. Let $A$ and $B^{*}$ be subnormal on $H$. Let $C$ be subnormal such that $N_{C}$ commutes with $N_{A}$ and also $D^{*}$ be subnormal such that $N_{D^{*}}$ commutes with $N_{B^{*}}$ respectively. Then

(i)

$$
\|A X D+C X B\|_{2} \geq\left\|A^{*} X D^{*}+C^{*} X B^{*}\right\|_{2}
$$

holds for every $X$ in $B(H)$. Equality in (**) holds for every $X$ in $B(H)$ when $A, B, C$ and $D$ are all normal.

(ii) If $X$ is an operator such that $A X D=C X B$, then $A^{*} X D^{*}=C^{*} X B^{*}$.

Proof. By Definition $1, N_{A}$ and $N_{C}$ are given by

$$
N_{A}=\left(\begin{array}{ll}
A & A_{12} \\
0 & A_{22}
\end{array}\right) \text { and } N_{C}=\left(\begin{array}{ll}
C & C_{12} \\
0 & C_{22}
\end{array}\right)
$$

acting on $H \oplus H$ whose restriction to $H \oplus\{0\}$ are $A$ and $C$ respectively and also $N_{B^{*}}$ and $N_{D^{*}}$ are given by the same reason as follows on $H \oplus H$;

$$
N_{B^{*}}=\left(\begin{array}{cc}
B^{*} & B_{12} \\
0 & B_{22}
\end{array}\right) \text { and } N_{D^{*}}=\left(\begin{array}{cc}
D^{*} & D_{12} \\
0 & D_{22}
\end{array}\right) \text {. }
$$

For $X$ acting on $H$, we consider $\left(\begin{array}{ll}X & 0 \\ 0 & 0\end{array}\right)$ acting on $H \oplus H \cdot\left\{N_{A}, N_{C}\right\}$ and $\left\{N_{D^{*}}^{*}, N_{B^{*}}^{*}\right\}$ are commuting pairs of normal operators on $H \oplus H$. Then, by Theorem $\mathrm{C}$, we have

$$
\begin{gathered}
\left\|\left(\begin{array}{ll}
A_{1} & A_{12} \\
0 & A_{22}
\end{array}\right)\left(\begin{array}{ll}
x & 0 \\
0 & 0
\end{array}\right)\left(\begin{array}{cc}
D & 0 \\
D_{12}^{*} & D_{22}^{*}
\end{array}\right)+\left(\begin{array}{ll}
C & C_{12} \\
0 & C_{22}
\end{array}\right)\left(\begin{array}{ll}
X & 0 \\
0 & 0
\end{array}\right)\left(\begin{array}{cc}
B & 0 \\
B_{12}^{*} & B_{22}^{*}
\end{array}\right)\right\|_{2} \\
=\left\|\left(\begin{array}{cc}
A^{*} & 0 \\
A_{12}^{*} & A_{22}^{*}
\end{array}\right)\left(\begin{array}{ll}
X & 0 \\
0 & 0
\end{array}\right)\left(\begin{array}{cc}
D^{*} & D_{12} \\
0 & D_{22}
\end{array}\right)+\left(\begin{array}{cc}
C^{*} & 0 \\
C_{12}^{*} & C_{22}^{*}
\end{array}\right)\left(\begin{array}{ll}
X & 0 \\
0 & 0
\end{array}\right)\left(\begin{array}{cc}
B^{*} & B_{12} \\
0 & B_{22}
\end{array}\right)\right\|_{2} ;
\end{gathered}
$$

that is,

$$
\left\|\left(\begin{array}{cc}
A X D+C X B & 0 \\
0 & 0
\end{array}\right)\right\|_{2}=\left\|\left(\begin{array}{cc}
A^{*} X D^{*}+C^{*} X B^{*} & A^{*} X D_{12}+C^{*} X B_{12} \\
A_{12^{*} X D^{*}+C_{12}^{*} X B^{*}} & A_{12}^{*} X D_{12}+C_{12}^{*} X B_{12}
\end{array}\right)\right\|_{2}
$$


so that

(3) $\|A X D+C X B\|_{2}^{2}=\left\|A^{*} X D^{*}+C^{*} X B^{*}\right\|_{2}^{2}+\left\|A^{*} X D_{12}+C^{*} X B_{12}\right\|_{2}^{2}$

$$
+\left\|A_{12}^{*} X D^{*}+C_{12}^{*} X B^{*}\right\|_{2}^{2}+\left\|A_{12}^{*} X D_{12}+C_{12}^{*} X B_{12}\right\|_{2}^{2}
$$

whence we have

$$
\|A X D+C X B\|_{2} \geq\left\|A^{*} X D^{*}+C^{*} X B^{*}\right\|_{2}
$$

which is the desired norm inequality (**). When $A, B, C$ and $D$ are all normal, then $A_{12}=0, B_{12}=0, C_{12}=0$ and $D_{12}=0$ in (3), so that equality in (**) holds and the proof is complete.

We remark that the sum of second, third and fourth of the right hand in (3) can be considered as a "perturbed terms" measures the deviation of subnormality from normality.

DEFINITION 2. Let $[S, T]$ * denote the following "*-commutator":

$$
[S, T]_{*}=S T-T S^{*} \text {; }
$$

this *-commutator is completely different from the usual commutator $[S, T]$.

DEFINITION 3. Let $S_{T}$ denote the positive square root of $\left[T^{*}, T\right]$ for the hyponormal operator $T$.

THEOREM 2. Let $A$ and $B^{*}$ be quasinormal on $H$. Let $C$ be $a$ quasinormal such that commutes with $A$ and satisfies $\left[A, S_{C}\right]_{*}=\left[C, S_{A}\right]_{*}$ and also let $D^{*}$ be a quasinomal such that commutes with $B^{*}$ and satisfies $\left[B^{*}, S_{D^{*}}\right]_{*}=\left[D^{*}, S_{B^{*}}\right]_{*}$ respectively. Then

(**)

$$
\|A X D+C X B\|_{2} \geq\left\|A^{*} X D^{*}+C^{*} X B^{*}\right\|_{2}
$$

holds for every $X$ in $B(H)$. Equality in (**) holds for every $X$ in $B(H)$ when $A, B, C$ and $D$ are all normal.

(ii) If $X$ is an operator such that $A X D=C X B$, then $A^{*} X D^{*}=C^{*} X B^{*}$.

Proof. Lei $A=U P$ be the polar decomposition of $A$, where $U$ is a partial isometry and $P$ is a positive operator such that $P^{2}=A^{\star} A$. A 
normal extension $N_{A}$ of $A$ can be written as follows [6, p. 308],

$$
N_{A}=\left(\begin{array}{cc}
A & S(A) \\
0 & A^{*}
\end{array}\right)
$$

acting on $H \oplus H$, where $S(A)=\left(I-U U^{*}\right) P$. Since $A$ is quasinormal, then $A=U P=P U$ [6, Problem 108]. As $U U^{*}$ is projection and $P$ commutes with $U$ and $U^{*}$, then

$$
\begin{aligned}
S(A) & =\left(I-U U^{*}\right) P=\left[\left(I-U U^{*}\right) P^{2}\right]^{\frac{1}{2}} \\
& =\left(P^{2}-U P U^{*} P\right)^{\frac{1}{2}}=\left(A^{*} A-A A^{*}\right)^{\frac{1}{2}}=S_{A} .
\end{aligned}
$$

Similarly normal extensions of $C, B^{*}$ and $D^{*}$ are also given as follows:

$$
N_{C}=\left(\begin{array}{cc}
C & S_{C} \\
0 & C^{*}
\end{array}\right), N_{B^{*}}=\left(\begin{array}{cc}
B^{*} & S_{B^{*}} \\
0 & B
\end{array}\right) \text { and } N_{D^{*}}=\left(\begin{array}{cc}
D^{*} & S_{D^{*}} \\
0 & D
\end{array}\right) \text {. }
$$

Hypotheses imply that $\left\{N_{A}, N_{C}\right\}$ and $\left\{N_{D^{*}}^{*}, N_{B^{*}}^{*}\right\}$ are pairs of commuting normal operators, so that the desired relations follow by the lemma.

COROLLARY 2. Let $A$ and $B^{*}$ be quasinormal on $H$. Let $C$ be $a$ normal commuting with $A$ and also $D$ be a normal commuting with $B$ respectively. Then

$$
\|A X D+C X B\|_{2} \geq\left\|A^{*} X D^{*}+C^{*} X B^{*}\right\|_{2}
$$

holds for every $X$ in $B(H)$. Equality in (**) holds for every $X$ in $B(H)$ when $A, B, C$ and $D$ are all normal.

(ii) If $X$ is an operator such that $A X D=C X B$, then $A^{*} X D^{*}=C^{*} X B^{*}$.

Proof. Take $N_{C}=\left(\begin{array}{ll}C & 0 \\ 0 & C\end{array}\right)$ in the proof of Theorem 2 since $C$ is normal. Then the hypothesis $C A=A C$ implies $C A^{*}=A^{*} C$ by the original Fuglede-Putnam theorem [1], [6], [7], [8], so that we have $C S_{A}^{2}=S_{A}^{2} C$; that is, $C S_{A}=S_{A} C$ holds, whence $N_{A}$ in the proof of Theorem 2 commutes with $N_{C}=\left(\begin{array}{ll}C & 0 \\ 0 & C\end{array}\right)$ since (4) holds. Similarly $N_{D^{*}}^{*}=\left(\begin{array}{cc}D^{*} & 0 \\ 0 & D^{*}\end{array}\right)$ commutes with $N_{B^{*}}^{*}$ in the proof of Theorem 2, so that the proof is complete by the 
Iemma.

REMARK 2. If we strengthen on $X$ to be in Hilbert-Schmidt class in Corollary 2, then we can relax quasinormality of the hypotheses on $A$ and $B^{*}$ to hyponormality and still retain the inequality; that is, just Corollary 1 .

COROLLARY 3. Let $A$ and $B^{*}$ be hyponormal satisfying $\left[A^{*}, S_{A}\right]_{*}=0$ and $\left[B, S_{B^{*}}\right]_{*}=0$ respectively. Let $C$ be a hyponormal which commutes with $A$ and satisfies $\left[C^{*}, S_{C}\right]_{*}=0$ and $\left[A, S_{C}\right]_{*}=\left[C, S_{A}\right]_{*}$ and also let $D^{*}$ be a hyponormal which commutes with $B^{*}$ and satisfies $\left[D, S_{D^{*}}\right]_{*}=0$ and $\left[B^{*}, S_{D^{*}}\right]_{*}=\left[D^{*}, S_{B^{*}}\right]_{*}$ respectively. Then

(i)

$$
\|A X D+C X B\|_{2} \geq\left\|A * X D^{*}+C * X B^{*}\right\|_{2}
$$

holds for every $X$ in $B(H)$. Equality in (**) holds for every $X$ in $B(H)$ when $A, B, C$ and $D$ are all normal.

(ii) If $X$ is an operator such that $A X D=C X B$, then $A^{*} X D^{*}=C^{*} X B^{*}$.

Proof. The hypotheses imply that $A, B^{*}, C$ and $D^{*}$ are all subnormal and $N_{A}=\left(\begin{array}{cc}A & S_{A} \\ 0 & A^{*}\end{array}\right)$ and similarly $N_{B^{*}}, N_{C}$ and $N_{D^{*}}$ are also given in the similar forms [4, Theorem 1]. As stated in the proof of Theorem 2, the hypotheses imply that $\left\{N_{A}, N_{C}\right\}$ and $\left\{N_{D^{*}}^{*}, N_{B^{*}}^{*}\right\}$ are pairs of commuting normal operators, so that the proof is complete by the lemma.

Can quasinormality be replaced by subnormality (or further hyponormality) in Theorem 2 and Corollary 2? Partial and modest answers to this question are cited in [2], [3], [9]. Theorem 1 is a modest result and Corollary 3 is in this direction. 


\section{References}

[1] S.K. Berberian, "Note on a theorem of Fuglede and Putnam", Proc. Amer. Math. Soc. 10 (1959), 175-182.

[2] S.K. Berberian, "Extensions of a theorem of Fuglede and Putnam", Proc. Amer. Math. Soc. 71 (1978), 113-114.

[3] Takayuki Furuta, "On relaxation of normality in the Fuglede-Putnam theorem", Proc. Amer. Math. Soc. 77 (1979), 324-328.

[4] Takayuki Furuta, Kyoko Matsumoto and Nobuhiro Moriya, "A simple condition on hyponormal operators implying subnormality", Math. Japon. 21 (1976), 399-400.

[5] Paul R. Halmos, "Shifts on Hilbert spaces", J. Reine Angew. Math. 208 (1961), 102-112.

[6] Paul R. Halmos, A Hilbert space problem book (Van Nostrand, Princeton, New Jersey; Toronto; London; 1967).

[7] C.R. Putnam, "On normal operators in Hilbert space", Amer. J. Math. 73 (1951), 357-362.

[8] M. Rosenblum, "On a theorem of Fuglede and Putnam", J. London Math. Soc. 33 (1958), 376-377.

[9] Joseph G. Stampfli \& Bhushan L. Wadhwa, "An asymmetric Putnam-Fuglede theorem for dominant operators", Indiana Univ. Math. J. 25 $(1976), 359-365$.

[10] Gary Weiss, "The Fuglede commutativity theorem modulo the HilbertSchmidt class and generating functions for matrix operators. I", Trans. Amer. Math. Soc. 246 (1978), 193-209.

[11] Gary Weiss, "The Fuglede commutativity theorem modulo the HilbertSchmidt class and generating functions for matrix operators. II", J. Oper. Theory 5 (1981), 3-16.

Department of Mathematics,

Faculty of Science,

Hirosaki University,

Bunkyo-Cho 3,

036 Aomori, Japan. 